\title{
Technischer Jugendschutz - Möglichkeiten und Grenzen nationaler Regulierung
}

\author{
Marc Liesching
}

\section{Einleitung}

Nach der Medienkonvergenz und der Digitalisierung der Medien Anfang des 21. Jahrhunderts entzieht die weltumspannende, dynamische und tendenziell anarchische Struktur des Internet zunehmend die Möglichkeit einer lückenlosen und systematisierten nationalen Regulierung in demokratischen Rechtsstaaten. Dies betrifft in besonderem Maße den Bereich des gesetzlichen Jugendmedienschutzes, der in Deutschland in seiner Komplexität und Ausdifferenzierung den Normsystemen anderer Staaten weitgehend entrückt ist. Klassische Jugendschutzinstrumente der ordnungsrechtlichen Aufsicht, aber auch der Selbstregulierung, können mit der Kommunikationsdynamik des Web 2.0 und auch der schieren Zahl von Milliarden von Medien- und Kommunikationsinhalten nicht schritthalten. Der Durchsetzung nationalen Jugendschutzrechts gegenüber Anbietern mit Sitz im EU- und im außereuropäischen Ausland sind zudem deutliche Grenzen gesetzt. Auch die Verantwortlichkeitsregeln des Telemediengesetzes (TMG) bzw. der E-CommerceRichtlinie (ECRL) schließen proaktive Überwachungspflichten der großen Medienintermediäre - insbesondere der Sozialen Medien - weitgehend aus.

Vor diesem Hintergrund wirft die wachsende Kluft zwischen dem materiell-rechtlichen Anspruch des deutschen Jugendschutzrechts einerseits und der faktischen Medienrealität der überwiegend freien $\mathrm{Zu}-$ gänglichkeit jugendgefährdender Medieninhalte andererseits Fragen nach der Erforderlichkeit einer Neuorientierung der Jugendschutzregulierung auf, ehe diese aufgrund der fehlenden tatsächlichen Durchsetzung Normakzeptanz bei Anbietern und Nutzern verliert und vor allem Kindern und Jugendlichen keinen Schutz mehr bietet.

Technische Lösungen - vor allem mit Blick auf die Fortentwicklung der KI-Technologien - werden daher zunehmend als möglicher Ausweg hin zu einem effektiven und möglichst lückenlosen Jugendmedienschutz diskutiert. Der nachfolgende Beitrag untersucht Möglichkeiten und Grenzen eines solchen technischen Jugendmedienschutzes. Dabei wird zunächst der aktuelle Stand der Regulierung technischen Jugendschutzes skizziert (hierzu II.), ehe auf die aus Sicht des Verf. bedeutende Frage der Möglichkeit automatisierter Erkennung jugendschutzwidriger Inhalte eingegangen wird (hierzu III.).
Im Anschluss werden - insbesondere rechtliche - Fragen im Zusammenhang mit einer möglichen Anbieterpflicht zur (technischen) Alterskennzeichnung diskutiert (hierzu IV.). Hieran schließt eine kursorische Darstellung möglicher Herausforderungen und Problemstellungen der Verknüpfung von Alterskennzeichen mit Restriktionen der Zugänglichkeit in Betriebssystemsoftware nutzerseitiger (internetfähiger) Empfangsgeräte (hierzu V.). Abschließend erfolgen Anmerkungen zum verfassungsrechtlichen Primat elterlicher Erziehung (hierzu VI.).

\section{Bisherige Regulierung technischen Jugendschutzes}

\section{1. Überblick}

Die bisherige Regulierung technischen Jugendschutzes bildet sich - abgesehen von den praktisch nicht mehr bedeutenden Bild-

Dr. Marc Liesching ist Professor für Medienrecht und Medientheorie an der Fakultät Medien der Hochschule für Technik, Wirtschaft und Kultur (HTWK) in Leipzig. Er ist u.a. Herausgeber des Gesetzeskommentars Liesching/Schuster: Jugendschutzrecht (5. Aufl. 2011, C.H. Beck Verlag).

träger-Automaten (vgl. § 12 Abs. 4 JuSchG), dem Erwachsenenversandhandel ( $§ 1$ Abs. 4 JuSchG) und Mitteilungen indizierter Telemedien für nutzerautonome Filter ( $\$ 24$ Abs. 5 JuSchG) - ganz überwiegend im Jugendmedienschutz-Staatsvertrag (JMStV) ab. Dabei basiert die Regelungssystematik auf einer generellen Pflicht für (Telemedien-)Anbieter, Zugangs- bzw. Wahrnehmungsbarrieren $\mathrm{zu}$ setzen, die verhindern, dass Kindern und Jugendlichen der betroffenen Altersgruppen die jugendgefährdenden bzw. entwicklungsbeeinträchtigenden Inhalte nicht bzw. »üblicherweise« nicht zugänglich sind

\section{AVS bei Pornografie und indizierten Inhalten in Telemedien}

Die strengsten Anforderungen gelten dabei bei unzulässigen jugendgefährdenden Telemedien, insbesondere Pornografie und indizierte Medieninhalte (vgl. § 4 Abs. 2 S. 2 JMStV). Hier muss "von Seiten des Anbieters sichergestellt" sein, dass entsprechende Angebote nur Erwachsenen zugänglich gemacht werden (geschlossene Benutzer- gruppe). Die technischen Anforderungen hieran sind sowohl nach der Spruchpraxis der Medienaufsicht als auch der Rechtsprechung recht hoch, da regelmäßig ein zweistufiges Verfahren der Identifikation und der Authentifikation gefordert wird, welche auf der Grundlage face-to-face verifizierter persönlicher Nutzerdaten basiert. ${ }^{1}$

Bereits hier zeigt sich in der Medienrealität die Kluft zwischen materiell-rechtlichem Normenanspruch und der in praxi freien Verfügbarkeit pornografischer Angebote über das Internet. Auch die jüngsten Bestrebungen der Landesmedienanstalten und ihrem Organ der Kommission für Jugendmedienschutz (KJM), gegen entsprechende große Anbieter mit Sitz in anderen EU-Mitgliedstaaten vorzugehen und ggf. auch auf Sperrverfügungen gegen Access-Provider ( $\$ 20$ Abs. 4 JMStV i.V.m. § 59 Abs. 4 RStV) zurückzugreifen, können nicht annähernd die freie Zugänglichkeit pornografischer Internetangebote in ihrer Gesamtheit eindämmen.

\section{Technische Mittel als Wahr- nehmungsbarriere}

Der Großteil der Medieninhalte, welche massenmedial vertrieben und von der Bevölkerung jedweden Alters rezipiert werden, hat entweder keine Jugendschutzrelevanz oder ist nur für bestimmte Altersstufen (6/12/16/18) von Minderjährigen entwicklungsbeeinträchtigend. ${ }^{2}$ Demgegenüber stellen die Medieninhalte, die von schwerwiegenderer Jugendschutzrelevanz sind und z.B. von der Bundesprüfstelle für jugendgefährdende Medien (BPjM) indiziert werden, eine Ausnahmeerscheinung dar. Dies gilt medienübergreifend sowohl für Rundfunksendungen als auch für Telemedien und für den Bildträgervertrieb.

Der Jugendmedienschutz-Staatsvertrag regelt im Abschnitt der »Allgemeinen Vorschriften « in § $5 \mathrm{JMStV}$ grundsätzlich übergreifend für alle »Angebote « im Sinne des § 3 Nr. $1 \mathrm{JMStV}$ die Anforderungen an die Verbreitung entwicklungsbeeinträchtigender Inhalte. Die Vorschrift des § 5 Abs. 1 S. 1 JMStV sieht für Anbieter entwicklungsbeeinträchtigender Rundfunksendungen und Telemedien einen allgemeinen Pflichtenstandard vor, der die Wahrnehmung entsprechender Inhalte durch betroffene Minderjährige »üblicherweise« verhindern soll.

Die Anbieterpflicht zur Wahrnehmungsbarriere kann dabei vom Anbieter auf verschiedene Weise umgesetzt werden. Neben 
Sendezeitbeschränkungen werden in $\S 5$ Abs. 3 JMStV ausdrücklich auch »technische Mittel« geregelt. Die Gemeinsamen Richtlinien der Landesmedienanstalten zur Gewährleistung des Schutzes der Menschenwürde und des Jugendschutzes ${ }^{3}$ definieren diese in Ziff. 3.3.1 als »Mittel im Rundfunk und in Telemedien, die von ihrer Wirksamkeit den Zeitgrenzen des $\S 5$ Abs. 3 Satz 1 Nr. 2 JMStV gleichzusetzen sind «.

\section{Jugendschutzprogramme}

Anbieter können als Nutzung »technischer Mittel« ihre entwicklungsbeeinträchtigenden Inhalte auch für ein Jugendschutzprogramm programmieren, das die entsprechende Alterskennzeichnung auslesen kann (§§ 5 Abs. 3 S. 1 Nr. 1, 11 JMStV). Bei der Auslesung von Alterskennzeichen genügt, dass die Inhalte vom Anbieter bewertet und kategorisiert werden, um dem Nutzer zu ermöglichen, durch Aktivierung eines bestimmten Erkennungs- und Kontrollprogramms auf seinem Rechner entwicklungsbeeinträchtigende Angebote differenziert nach Altersstufen herauszufiltern. Die Letztverantwortung verbleibt hier stets bei den Eltern. ${ }^{4}$ Im Zusammenhang mit der durch die KJM erfolgten Anerkennung des Programms JusProG ${ }^{5}$ hat sich faktisch das XML-Format mit der Hinterlegung einer age-de.xml-Datei durch den Webseitenbetreiber etabliert. ${ }^{6}$ Allerdings ist der xmlStandard nicht bei allen proprietären Systemen einsetzbar.

\section{Automatische Erkennung jugend- schutzwidriger Inhalte?}

\section{Gesetzliche Berücksichtigung de lege lata}

Die Regelung zu Jugendschutzprogrammen in $\S 11 \mathrm{JMStV}$ sieht seit ihrer Neufassung durch den 19. RfÄndStV als Eignungsvoraussetzungen auch ausdrücklich vor, dass die Programme »eine dem Stand der Technik entsprechende Erkennungsleistung aufweisen $«$.

Die Landesgesetzgeber verstehen unter dem »Stand der Technik« unter Rückgriff auf die immissionsschutzrechtliche Verwendung des Begriffs den »Entwicklungsstand fortschrittlicher Verfahren, Einrichtungen oder Betriebsweisen, der die praktische Eignung der Maßnahme im Hinblick auf die angestrebten Ziele insgesamt gesichert erscheinen lässt « ${ }^{7}$ Durch die offene und weithin unbestimmte Formulierung soll klargestellt werden, dass sich Jugendschutzprogramme an den stetig fortschreitenden technischen Entwicklungen messen lassen müssen ${ }^{8}{ }^{8}$ Mithin ergeben sich aus der Amtlichen Begründung weder Klarstellungen noch Konkretisierungen.

\section{Grenzen automatisierter »Erkennung “}

Ungeachtet der Fortschritte bei der Entwicklung von KI-Technologien insbesondere im Bereich der automatisierten Bilderkennung ergeben sich erhebliche Problemstellungen bei einer Anwendung im Jugendmedienschutz, soweit hiermit auch bei »erkannten« Inhalten automatische Verbreitungsrestriktionen verknüpft werden sollen. Denn im Unterschied zur Erkennung von »Katzenbildern« oder »menschlichen Gesichtern« kann die Identifikation »entwicklungsbeeinträchtigender« Inhalte nicht nur auf vorgegebenen Datensätzen spezifischer bestimmter Merkmale fußen. Vielmehr bedarf es einer wertend prognostischen Beurteilung, ob ein bestimmter Inhalt geeignet ist, Kinder und Jugendliche zu beeinträchtigen - und dies auch noch abgestuft nach den Altersgruppen 0, 6, 12, 16, 18 .

Schon angesichts dessen, dass selbst professionalisierte menschliche Entscheidungsträger im Jugendmedienschutz über verwaltungsgerichtliche Instanzen hinweg bei der Frage »ab 6« oder »ab 12« (oder im Rahmen von »ab $12 «$ ob Tagesprogramm oder Hauptabendprogramm) keinen Bewertungskonsens erzielen können, ${ }^{9}$ erscheint fraglich, wie automatisierte Bewertungsprozesse eine annähernd korrekte »Erkennung « erreichen können. Denn im Grunde geht es gerade nicht (nur) um die »Erkennung « - wie der Wortlaut des $\S 11 \mathrm{JMStV}$ suggeriert - sondern um die »Bewertung « der Entwicklungsbeeinträchtigung eines Inhaltes.

Schon die aktuell zum Teil erprobten, zum Teil in der frühen Umsetzung befindlichen Bewertungssysteme IARC und das SKS-Modell der FSK basieren gerade nicht auf automatisierten »technisch-individualisierten « Bewertungen, sondern auf simplifizierten »Wenn-Dann« Logiken auf der Grundlage eines vom Anbieter auszufüllenden Fragebogens, der bereits implizit Wertungsparameter abfragt. Davon zu unterscheiden ist aber gerade die durch $\S 11$ Abs. 1 JMStV implizierte Vorstellung, eine artifizielle KI-Entität könne erkennen, ob z.B. Gewalt »verherrlichend oder verharmlosend dargestellt wird, »rückwärtsgewandte Rollenklischees « kolportiert werden, bestimmte Bevölkerungsgruppen in Texten »diskriminiert « werden und ein entsprechend "problematisches Wertebild« von Kindern einer bestimmten Altersgruppe "nicht hinreichend entschlüsselt und übernommen werden kann«, »höhere Altersgruppen« aufgrund der »Realitätsferne« der Darstellung sich »distanzieren « können, eine besondere »Alltagsnähe « oder eine »kinderaffine Gestaltung « der Inhalte Kinder besonders anspricht, eine »klare Gut-Böse-Zeichnung vorhanden ist, welche Kindern die Einordnung erleichtert oder ein »Berichterstattungsinteresse oder satirische Überspitzung zu einer abweichenden Gesamtbewertung führen müssen« etc.

So sehr es angesichts der einleitend skizzierten Kluft des Jugendschutzanspruchs und der Medienrealität auch politisch gewollt sein mag, eine zutreffende und die komplexen Anforderungen jugendschutzrechtlich sachgerechter Inhalte-Bewertungen berücksichtigende rein technische »Erkennung « entwicklungsbeeinträchtigender Inhalte, möglichst noch nach fünf Altersstufen, ist der Realität der aktuellen KI-Entwicklung entrückt.

Vor diesem Hintergrund fokussiert sich die aktuelle - auch rechtspolitische - Diskussion auf die Möglichkeit einer (obligatorischen) Jugendschutzbewertung von Angebotsinhalten durch Menschen. Der darauf basierende Gedanke ist einfach: Sind alle Angebotsinhalte vom Anbieter mit einer Alterskennzeichnung versehen, kann diese auf technischem Wege - z.B. durch Jugendschutzprogramme oder Betriebssysteme auf Empfangsgeräten - erkannt werden. Aufgrund der Erkennung kann entsprechend den Voreinstellungen in der Software der betreffende Inhalt entweder blockiert oder angezeigt werden.

\section{Alterskennzeichnungspflicht für Anbieter?}

\section{Möglichkeiten der Regulierung}

\section{a) Aktuelle Rechtslage}

Die bloße fakultative Option einer Alterskennzeichnung von Inhalten durch Anbieter ist bereits seit 2003 Teil des jugendschutzregulativen Ansatzes. Denn seit jeher sieht § 5 i.V.m. § $11 \mathrm{JMStV}$ die Möglichkeit für Anbieter vor, ihrer Pflicht zur Wahrnehmungsbarriere (siehe oben) dadurch nachzukommen, dass sie entwicklungsbeeinträchtigende Inhalte für eine Altersstufe kennzeichnen und entsprechend für ein anerkanntes bzw. als "geeignet « positiv bewertetes Jugendschutzprogramm labeln. Allerdings erfolgt eine Umsetzung im Telemedienbereich bei weitem nicht flächendeckend. Zum einen ist davon auszugehen, dass eine Vielzahl von Anbietern im Ausland das Pflichtenregime des deutschen Jugendmedienschutzes in $\S 5 \mathrm{JMStV}$ noch nicht einmal kennen.

Zum anderen sieht der Jugendmedienschutz-Staatsvertrag selbst zahlreiche Alternativen vor, wie der Anbieter seiner Jugendschutzpflicht im Bereich entwicklungsbeeinträchtigender Angebote nachkommen kann. Für Inhalte im Bereich 0/6/12 genügt es ohnehin schon, dass solche Inhalte gemäß $§ 5$ Abs. $5 \mathrm{JMStV}$ »getrennt von für Kinder bestimmten Angeboten verbreitet«, was in der Regel schon von vorneherein der Fall ist. Diese Inhalte können also auch nach 
dem Jugendschutzrecht frei von einer Alterskennzeichnung oder Wahrnehmungsbeschränkungen zugänglich gemacht werden. Für 16/18 Inhalte genügt jugendschutzrechtlich ein Rating bzw. auch ein »Overrating « durch den XML-Code »18 « für JusProg, alternativ kann man aber auch eine Verbreitungszeitbeschränkung (23:00-6:00) oder alternative Barrieren wie eine Kreditkartenabfrage oder die Eingabe einer Personalausweisziffer vorsehen, ohne zusätzlich eine Alterskennzeichnung durchzuführen. Speziell für große OnlineFilm- und Spielplattformen soll sich dies freilich mit dem aktuell im parlamentarischen Abstimmungsprozess befindlichen § 14a JuSchGÄndG-E ändern.

\section{b) Optionen künftiger regulativer Durchsetzung}

Wollte man als Grundlage einer möglichst flächendeckenden technischen Auslesung künftig Anbieter breit zu Alterskennzeichnungen ihrer Inhalte anhalten, so käme entweder eine unmittelbar geregelte gesetzliche Pflicht zur Kennzeichnung aller Anbieterinhalte nach Altersstufen in Betracht. Oder der (Landes-)Gesetzgeber schafft zwar keine direkte Pflichtenregelung zur Alterskennzeichnung, normiert dafür aber strenge default-Vorgaben für Endgeräte (Betriebssysteme), welche bei Anbietern faktisch einer Verpflichtung gleichkommen, wenn sie mit ihren Inhalten wahrnehmbar bleiben wollen. Beide regulativen Ansätze evozieren freilich erhebliche rechtliche Frage- und Problemstellungen, welche nachfolgend ohne Anspruch auf Vollständigkeit skizziert werden.

\section{Europarechtliche Grenzen}

Der Ansatz eines möglichst flächendeckenden Jugendmedienschutzes stößt - wie bereits in der Einleitung angedeutet - im Falle des Versuchs einer nationalgesetzlich verpflichtenden Regelung auch für ausländische Unternehmen zumindest im EU-Raum auf enge Grenzen, die insbesondere durch die Bestimmungen zum Herkunftslandprinzip in Art. 3 der E-Commerce-Richtlinie (ECRL) ${ }^{10}$ und auch Art 3 AVMD-RL bedingt sind.

Gemäß Art. 3 Abs. 2 ECRL dürfen die Mitgliedstaaten den freien Verkehr von Diensten der Informationsgesellschaft aus einem anderen Mitgliedstaat nicht aus Gründen einschränken, die in den koordinierten Bereich fallen. Der EuGH hat bestätigt, dass es das hierin verankerte Herkunftslandprinzip vorbehaltlich der engen Ausnahmen nicht zulässt, »dass der Anbieter eines Dienstes des elektronischen Geschäftsverkehrs strengeren Anforderungen unterliegt, als sie das im Sitzmitgliedstaat dieses Anbieters geltende Sachrecht vorsieht «.11
Maßnahmen durch EU-Empfangsstaaten sind nur im Rahmen der sehr engen Ausnahmen nach Art. 3 Abs. 4-6 ECRL lediglich »im Hinblick auf einen bestimmten Dienst der Informationsgesellschaft « sowie nur zur Verfolgung besonderer Schutzziele wie z.B. der »öffentlichen Ordnung, insbesondere Verhütung, Ermittlung, Aufklärung und Verfolgung von Straftaten, einschließlich des Jugendschutzes« möglich. Dabei muss der durch die Maßnahmen betroffene »bestimmte Dienst der Informationsgesellschaft" eines der Schutzziele »beeinträchtigen« oder »eine ernsthafte und schwerwiegende Gefahr einer Beeinträchtigung dieser Ziele« darstellen. Darüber hinaus müssen die Maßnahmen auch angemessen im Sinne einer Verhältnismäßigkeit in Bezug auf die Schutzziele sein. ${ }^{12}$

Eine gesetzliche Verpflichtung etwa zur Etablierung »technischer Programme« in Betriebssystemen, welche z.B. nicht gekennzeichnete Telemedien generell als »ab 18 « labeln sollen und diese in der DefaultEinstellung nicht zugänglich machen, stellte dabei eine Maßnahme dar, die geeignet wäre, den freien Verkehr von Diensten der Informationsgesellschaft aus anderen Mitgliedstaaten erheblich einzuschränken, soweit nicht sichergestellt wird, dass keine Telemedieninhalte von Anbietern mit Sitz in einem anderen EU-Mitgliedstaat von der betriebssystembedingten Zugangsbeschräkung betroffen werden.

\section{Verfassungsrechtlicher Rahmen des Art. 5 Abs. 1 GG}

a) Wesentlicher Bezugspunkt der Grundrechte des Art. 5 Abs. 1 S. 1 und 2 GG ist die Gewährleistung des freien Austauschs von Informationen und Meinungen zur öffentlichen Meinungsbildung. ${ }^{13}$ Die umfassend geschützte Meinungsäußerungsfreiheit wird dabei durch jede staatliche Maßnahme beeinträchtigt, die die Meinungsäußerung und -verbreitung behindert. Auch die nach Abs. 1 2. Hs. geschützte Informationsfreiheit ist aufgrund des weiten Wortlauts (»ungehinderte « Unterrichtung aus allgemein zugänglichen Quellen) bereits dann durch staatliche Maßnahmen betroffen, wenn diese nur eine Verzögerung der Informationsaufnahme bewirken. ${ }^{14}$ Die gesetzgeberische Behinderung der Beschaffung und Entgegennahme von Informationen aus dem Internet über internetfähige Hardware greift daher in den Schutzbereich der Informationsfreiheit ein. ${ }^{15}$ Darüber hinaus besteht ein verfassungsrechtlicher Mediendistributionsschutz im Rahmen des Art. 5 Abs. 1 S. 2 GG. ${ }^{16}$

b) Eine regulative Kombination einer Voreinstellung »ab 18« z.B. in Betriebssystemen von Empfangsgeräten einerseits mit einer automatischen Behandlung "ab 18 « aller nicht von Anbietern gekennzeichne- ten Sendungen und Telemedien andererseits führte zu einer Blockierung der allermeisten geäußerten Meinungen, Informationen und sonstigen meinungsbildenden Inhalte. Auch wenn diese nachträglich seitens des Nutzers durch Änderung der Einstellungen aufgehoben werden kann, z.B. durch einen Altersnachweis (gegenüber wem auch immer), blieben die Einschränkungen der verfassungsrechtlichen Kommunikationsund Informationsfreiheit massiv.

Überdies würden Telemedienanbietern die bisher in $\S 5$ Abs. $3 \mathrm{JMStV}$ vorgesehenen Alternativen von Wahrnehmungsbarrieren (technische, oder sonstige Mittel, Verbreitungszeitbeschänkungen) faktisch genommen. Denn wenn Betriebssysteme aufgrund obligatorischer »technischer Programme « solche nach $\S 5$ Abs. $3 \mathrm{JMStV}$ angebotenen Inhalte gar nicht als jugendschutzkonform erkennen können, würden sie blockiert und sind für Nutzer in den Grundeinstellungen nicht wahrnehmbar, obwohl sie gerade die Vorgaben des $\S 5$ Abs. 3 JMStV (z.B. KJM-anerkanntes technisches Mittel, ${ }^{17}$ Sendezeitbeschränkung, Trennung von 6/12 Inhalten und Kinderangeboten i.S.d. § 5 Abs. $5 \mathrm{JMStV}$ ) explizit und willentlich beachten.

c) Aufgrund solcher Eingriffe in den Schutzbereich von Art. 5 Abs. 1 GG müssten sich entsprechende gesetzliche Bestimmungen an den Vorgaben zu Schrankenregelungen nach Art. 5 Abs. 2 GG messen lassen. Insbesondere wäre nach den Vorgaben des BVerfG zu klären, ob die Güterabwägung zu einem Vorrang des Schutzes des Rechtsguts führt, dem das allgemeine Gesetz bzw. die Jugendschutzvorschrift ${ }^{18}$ dient. $^{19}$ Es muss deshalb seinerseits im Lichte des beschränkten Grundrechts ausgelegt ${ }^{20}$ und unter Beachtung des Verhältnismäßigkeitsgrundsatzes angewendet werden. ${ }^{21}$

Die Kombination einer BetriebssystemVoreinstellung als minderjährig »ab 18 « mit einem Labeling aller nicht gekennzeichneten Inhalte als »ab 18 « führte indes $\mathrm{zu}$ einem Blockieren des Zugangs zu den allermeisten Informationsinhalten über internet- und rundfunkfähige Empfangsgeräte in der Default-Einstellung auch für erwachsene NutzerInnen. Dies ist schon nicht erforderlich, da mit der Möglichkeit der nur fakultativen Aktivierung von Einstellungen in Eltern-Eigenverantwortung ein milderes Mittel zur Verfügung steht, welches die Landesgesetzgeber seit 2003 mit Blick auf § 11 JMStV (Jugendschutzprogramme) als geeignetes Mittel ansehen.

Mögliche gesetzliche Einschränkungen einer pauschalen Minderjährigkeitsvermutung der Erwerber internetfähiger Empfangsgeräte (z.B. bei entsprechender Voreinstellung im Betriebssystem) wären dabei auch vom elterlichen Erziehungsprimat ${ }^{22}$ und nicht mehr von dem nach Art. 6 
Abs. 2 GG verfassungsrechtlich legitimierten staatlichen Schutzauftrag gedeckt. Denn nur technische Lösungen zur Zugangsbeschränkung, welche die Letztverantwortung der Implementierung und Erstinitiierung den Eltern überlassen - so etwa bei Jugendschutzprogrammen nach $\S 11 \mathrm{JMStV}$-tragenden Vorgaben des BVerfG zur Begrenzung des staatlichen Wächteramts durch den verfassungsrechtlich manifestierten Vorrang der Eltern im Sinne der Eigenständigkeit und Selbstverantwortlichkeit bei der Erziehung der Kinder Rechnung. ${ }^{23}$

\section{Beachtung der Verantwortlichkeits- regeln}

Eine proaktive Alterskennzeichnungspflicht wäre aufgrund der Verantwortlichkeitsregelungen insbesondere des § $10 \mathrm{TMG}$ in den marktrelevanten Medienplattformen der Intermediäre und Sozialen Medien kaum durchsetzbar. Denn Host-Provider sind vor konkreter Kenntnis von Inhalten der Uploader gar nicht rechtlich verantwortlich für die Einhaltung des Jugendschutzes. Insoweit könnte eine Alterskennzeichnungspflicht allenfalls dann begründet sein, wenn der Plattformbetreiber z.B. aufgrund einer Beschwerde von einem jugendschutzrelevanten Inhalt Kenntnis genommen hat. Auch dann bliebe es dem Anbieter freilich unbenommen, statt einer Alterskennzeichnung den Inhalt einfach zu löschen oder die Zugänglichkeit jedenfalls für den deutschen Empfangsbereich komplett zu blockieren.

Auch soweit in einem nationalen Jugendschutzgesetz normiert würde, dass z.B. Betriebssysteme nicht gekennzeichnete Inhalte »selbst prüfen« müssten, ergäbe sich - ungeachtet der Umsetzbarkeit - ein Widerspruch zu den europarechtlichen und bundesgesetzlich umgesetzten Verantwortlichkeitsabstufungen nach Art. 12-15 ECRL und § 7-10 TMG. Insbesondere sind Diensteanbieter im Sinne der $\S \S 8$ bis $\S 10$ TMG nicht verpflichtet, die von ihnen übermittelten oder gespeicherten Informationen zu überwachen oder nach Umständen zu forschen, die auf eine rechtswidrige Tätigkeit hinweisen. Eine Auslegung des nationalen Rechts, die im Ergebnis dazu führen würde, eine anlassunabhängige generelle (»proaktive«) Überwachungspflicht $\mathrm{zu}$ statuieren oder vorauszusetzen, wäre richtlinienwidrig und damit unzulässig. ${ }^{24}$

Zwar stellen Betriebssysteme gerade keine Telemedien dar, sodass Hersteller und Vertreiber solcher Betriebssysteme als potentielle Normadressaten auch keine Diensteanbieter i.S.d. 7 Abs. 2 TMG sind. Jedoch wird die auf Telemedien bezogene Verantwortlichkeitsregulierung unterminiert, wenn die von den Inhalten noch weiter als AccessProvider ( 88 TMG) entfernt stehenden Betriebssystemhersteller faktisch zu einer proaktiven Inhaltsprüfung von Telemedien mit Blick auf eine etwaige Entwicklungsbeeinträchtigung verpflichtet würden. Hierdurch wäre das Pflichten- und Haftungssystem in Bezug auf (fremde), zugangsvermittelte Diensteinhalte vollumfänglich desavouiert.

\section{Systemkongruenz zum JMStV}

\section{a) Offene Optionen für Wahrnehmungs- barrieren}

Zudem könnten Regelungen einer Alterskennzeichnungspflicht zumindest faktisch zu einer Negierung der bisherigen alternativ für Anbieter möglichen Jugendschutzmaßnahmen nach § 5 Abs. $3 \mathrm{JMStV}$ führen. Insbesondere werden Angebote, welche ihre Inhalte jugendschutzkonform nur zu einer zulässigen Verbreitungszeit (§ 5 Abs. 4 JMStV) zugänglich machen oder ein technisches Mittel vorschalten (z.B. KJManerkannte Tools zur Prüfung von Personalausweisnummern ${ }^{25}$ ) als Folge einer Neuregelung z.B. auf Betriebssystem-Basis auf keinem internetfähigen Empfangsgerät mehr wahrnehmbar sein. Denn »technische Programme " in Betriebssystemen könnten wahrscheinlich solche nach $\S 5$ Abs. 3 JMStV angebotenen Inhalte gar nicht als jugendschutzkonform erkennen, sofern nicht auch zusätzlich eine Alterskennzeichnung vorgenommen wird, welche wiederum alle anderen möglichen Maßnahmen des § 5 Abs. 3 JMStV überflüssig macht. Faktisch würde also eine Alterskennzeichnung für Betriebssysteme, Jugendschutzprogramme und Medienintermediäre alternativlos, sofern der Anbieter mit seinen Inhalten noch von einer relevanten Gruppe von NutzerInnen wahrgenommen werden will.

Auch Angebote, die entwicklungsbeeinträchtigend für Altersgruppen »unter 6 « bzw. »unter 12 « Jahren sind und die gemäß $\S 5$ Abs. $5 \mathrm{JMStV}$ lediglich getrennt von Kinderangeboten verbreitet werden müssen, um den Anforderungen des $§ 5$ Abs. 1 JMStV zu genügen, würden bei normierten Voreinstellungen im Betriebssystem für Nutzer möglicherweise geblockt werden. Die bisherigen im Gesetz (dann rein deklaratorisch) stehenden Maßnahme-Alternativen für Anbieter (Sendezeitbeschränkung, technische oder sonstige Mittel, Trennung von Kinderangeboten bei 6/12 Inhalten) wären mithin rechtstatsächlich gegenüber der faktischen Alterskennzeichnungspflicht ihrer Inhalte keine Option mehr für Anbieter.

\section{b) Berichterstatterprivileg des $\S 5$ Abs. 6 JMStV}

In Bezug auf journalistisch-redaktionelle Angebote sind rechtssystematisch die Einschränkungen des $§ 5$ Abs. 6 und 7 JMStV zu berücksichtigen. Danach gilt die Anbieterpflicht für Wahrnehmungsbarrieren von vorneherein nicht für Nachrichtensendungen, Sendungen zum politischen Zeitgeschehen im Rundfunk und "vergleichbare Angebote bei Telemedien", es sei denn, es besteht kein berechtigtes Interesse an dieser Form der Darstellung oder Berichterstattung. Bei Angeboten, die Inhalte periodischer Druckerzeugnisse in Text und Bild wiedergeben, gelten die Beschränkungen des $§ 5$ Abs. 1 JMStV erst dann, wenn die KJM gegenüber dem Anbieter festgestellt hat, dass das Angebot entwicklungsbeeinträchtigend ist.

Nach § 5 Abs. 7 JMStV wird aufgrund des Berichterstattungsinteresses und im Rahmen des verfassungsrechtlichen Schutzes der Meinungsbildungsfunktion der Medien im Einzelfalleine Verbreitung von Nachrichteninhalten gewährleistet, auch wenn diese im konkreten Einzelfall entwicklungsbeeinträchtigend sind (Berichterstattungsprivileg). ${ }^{26}$ Insoweit ist unklar, wie dies in einem binären technischen Schutzkonzept umzusetzen ist. Denn hiernach wäre nur die Altersstufe nach Entwicklungsbeeinträchtigungsgrad mitzuteilen, was z.B. zu einer Blockierung des Zugangs im voreingestellten Betriebssystem führt und somit das Berichterstattungsprivileg des $\S 5$ Abs. 7 JMStV unterminiert. Entsprechende Schwierigkeiten können in Bezug auf $\S 5$ Abs. 8 JMStV bei von KJM-Beurteilung im Einzelfall abhängiger Jugendschutzrelevanz der Entwicklungsbeeinträchtigung auftreten.

\section{Weitere Problemstellungen einer Betriebssystem-Regulierung}

\section{Gesetzgebungskompetenz}

a) Bisher unklar erscheint die Gesetzgebungskompetenz für eine etwaige Jugendschutzregulierung von Betriebssystemen rundfunk- und internetfähiger Geräte. Die umfassende Gesetzgebungskompetenz des Bundes für den Bereich des Jugendschutzes einschließlich des gesamten Medienbereichs ergibt sich aus den Verfassungsbestimmungen des Art. 72, 74 Abs. 1 Nr. 1 GG, welche dem Bund die Regelungsbefugnis für den Bereich »Strafrecht « im Wege der konkurrierenden Gesetzgebung zuweisen. ${ }^{27}$ Daneben ergibt sich die Kompetenzzuweisung aus Art. 74 Abs. 1 Nr. 7 GG, welche die Regelungsmaterie der »öffentlichen Fürsorge « zum Gegenstand hat. ${ }^{28}$ Der Bund hat im Rahmen der konkurrierenden Gesetzgebung seine Kompetenz zur Normierung des Jugendmedienschutzes - gerade auch in Bezug auf die elektronischen Informationsund Kommunikationsdienste und die hiermit im Sachzusammenhang stehenden Bereiche - wahrgenommen. ${ }^{29}$

Schon auf der Grundlage der Bund-Länder-Vereinbarungen 2002 ist mit Inkraft- 
treten des JuSchG den Ländern neben dem Rundfunk nur eingeschränkt die Regulierung in Bezug auf »Telemedien« verblieben. Neben Rechtsfolgen zu indizierten Telemedien (§ 16 JuSchG) betrifft dies ausweislich der JuSchG-Gesetzesbegründung abschließend nur »Regelungen über Jugendschutzbeauftragte, Freiwillige Selbstkontrolle und Filterprogramme sowie jugendbeeinträchtigenden Telemedien ${ }^{30}{ }^{30}$ Hardwaregebundene Betriebssysteme als Regelungsgegenstand sind aber weder Telemedien noch sind sie Filterprogramme.

DieWahrnehmungder Regulierungskompetenz für den Jugendschutz begründet eine Sperrwirkung nach Art. 72 Abs. 1 GG, soweit der Bund von seiner Gesetzgebungskompetenz Gebrauch gemacht hat. Dabei ist gerade unerheblich, dass der Bund im Rahmen des JuSchG keine Regelungen zu HardwareBetriebssystemen getroffen hat. Denn insoweit kann die Sperrwirkung nach der Rechtsprechung des BVerfG gerade auch durch erkennbaren, absichtsvollen Regelungsverzicht begründet werden. ${ }^{31} \mathrm{Ob}$ der Gebrauch, den der Bund von einer Kompetenz gemacht hat, abschließend ist, muss aufgrund einer Gesamtwürdigung des betreffenden Normenkomplexes festgestellt werden. ${ }^{32}$

Die Sperrwirkung für die Länder ist aufgrund der Kompetenzwahrnehmung durch den Bund mit der Fassung des Jugendschutzgesetzes sowie den zugrunde liegenden und in den JuSchG-/JMStV-Gesetzesbegründungen referenzierten Bund-LänderEckpunktevereinbarungen hinreichend erkennbar. Danach regeln die Länder neben dem Rundfunk den Jugendschutz »in allen elektronischen Medien ${ },{ }^{33}$ hingegen der »Bund den Jugendschutz bei den OfflineMedien und den Jugendschutz außerhalb des Medienbereichs «. ${ }^{34}$ Hardwarebezogene Betriebssysteme sind nach den Legaldefinitionen in § 1 Abs. 2 und 3 JuSchG weder Trägermedien noch Telemedien. ${ }^{35}$ Sie sind vielmehr überhaupt keine Medien im jugendschutzrechtlich inhaltsbezogenen Sinne, sondern außerhalb des Medienbereichs auf die Funktion von Hardware ausgerichtet.

Die Gesetzgebungskompetenz der Länder beschränkt sich auch nach den Ausführungen der Länder in der Begründung zum JMStV auf den »Jugendschutz in Telemedien« (und Rundfunk) bzw. »materielle Regelungen für Anbieter von Rundfunk und Telemedien $\ll{ }^{36}$ Dies entspricht auch dem seitens des Bundes explizit geäußerten Regelungsverzicht auf Teilnormierungsbereiche ausschließlich im Sektor der »Telemedien «.

\section{Verfassungsrechtliche Position der Betriebssystem-Hersteller}

Durch entsprechende obligatorische Jugendschutzregelungen für Geräte- und Betriebssystemsoftware-Hersteller könnte auch erheblich in deren Grundrechtspositionen eingegriffen werden. Denn insoweit werden grundlegende Vorgaben in Produkt- und Nutzungseigenschaften marktrelevanter Medienempfangsgeräte gemacht, welche enorme Einschränkungen der Nutzung der Produkte mit sich bringen könnten. Überdies ist auch der wirtschaftliche Aufwand einschließlich des Einsatzes von Personalressourcen für die tiefgreifende Anpassung von Softwarestruktur bei rundfunk- und internetfähigen Endgeräten (z.B. Smartphones, Laptops, Spielkonsolen, SmartTVs, Set-top-Entertainment-Geräte, PC-Betriebssysteme) zu berücksichtigen.

Dies berührt den Schutzbereich nach Art. 12, 14 GG, der schon aufgrund der fehlenden Medienanbietereigenschaft von Geräteherstellern und Betriebssystemsoftware-Herstellern nicht gegenüber Art. 5 GG subsidiär ist. ${ }^{37}$ Eine Rechtfertigung solcher Eingriffe erscheint vorliegend fraglich, zumal die Geräte- und BetriebssystemsoftwareHersteller selbst nicht jugendgefährdende Sendungen und Telemedien verbreiten und mithin eine Verhältnismäßigkeit ${ }^{38}$ tiefgreifender Restriktionen eine rechtspolitische Herausforderung darstellen. Im Rahmen der Verhältnismäßigkeitsprüfung ist ebenso zu berücksichtigen, dass Geräte- und Betriebssystemsoftware-Hersteller selbst gar nicht für entwicklungsbeeinträchtigende Telemedien verantwortlich sind. Sie stehen schon aufgrund der regelmäßig fehlenden Anbietereigenschaft außerhalb des rechtlichen Haftungssystems und sind hinsichtlich ihrer Funktion bei der Zugangsvermittlung noch weiter von inkriminierten Inhalten entfernt als etwa Access-Provider i.S.d. $§ 8$ TMG. $^{39}$

\section{Grenzen der Vollzugsmöglichkeiten}

Eine regulatorische Okkupation von Betriebssystemen ginge - ungeachtet der bereits angesprochenen Frage der Gesetzgebungskompetenz - darüber hinaus zwingend mit einer Erweiterung des bisher auf Rundfunk- und Telemedienanbieter beschränkten Anwendungsbereichs des JMStV einher. Sofern die Maßnahmenkompetenz der Landesmedienanstalten und ihres Organs KJM auf Anbieter von Telemedien und Rundfunk beschränkt bliebe, wären Betriebssystemlösungen nicht im Vollzug durchsetzbar.

Ungeachtet dessen bestünde die zentrale Herausforderung einer Umsetzung nationaler Jugendschutz-Betriebssystem-Regelungen darin, dass nahezu alle marktrelevanten Hersteller/Entwickler ihren Sitz nicht in der Bundesrepublik haben. Hier ergeben sich die bereits skizzierten Fragestellungen der Unionsrechtskonformität nationaler Maßnahmen gegen Anbieter mit Sitz in einem anderen EU-Mitgliedstaat. Hinzu kommen weltweit und insbesondere mit Blick auf Unternehmen in den USA Fragestellungen des Vollzugs deutscher verwaltungsrechtlicher Jugendschutzpflichten. Etwaige Bußgeldbewehrungen unterliegen darüber hinaus schon der Frage der Anwendbarkeit deutschen Ordnungswidrigkeitenrechts nach $\S \S 5,7$ OWiG.

\section{Technischer Jugendschutz - Primat elterlicher Erziehung}

Sind die Landesgesetzgeber 2003 bei Jugendschutzprogrammen ( $§ 11 \mathrm{JMStV}$ ) noch davon ausgegangen, dass hinsichtlich der Aktivierung solcher Programme zur Auslesung von Alterskennzeichen »die Letztverantwortung der Eltern oder Erziehungsberechtigten bestehen « bleibt, ${ }^{40}$ würden obligatorische Anbieterpflichten einer zwingenden Alterskennzeichnung - verknüpft mit geräteseitigen Grundeinstellungen im Betriebssystem - wesentlich weitergehen. Auch mit Blick auf die Schrankenregelung des Art. 5 Abs. 2 GG bedarf es indes bei technischen Jugendschutzlösungen einer grundsätzlichen Klärung der verfassungsrechtlichen Legitimität von gesetzlich angeordneten Jugendschutzmaßnahmen im Vorgriff auf konkrete elterliche Erziehungsentscheidungen.

Das BVerfG leitet den Verfassungsrang des Jugendschutzes vor allem aus dem in Art. 6 Abs. 2 S. 1 GG verbrieften elterlichen Erziehungsrecht $a b .{ }^{41}$ Danach sind $»$ Pflege und Erziehung der Kinder das natürliche Recht der Eltern und die zuvörderst ihnen obliegende Pflicht«. Dieser prinzipielle Elternvorrang basiert auf der Grundannahme, dass diejenigen, die einem Kind das Leben schenken, auch von Natur aus dazu berufen und bereit sind, die Verantwortung für dessen Pflege und Erziehung zu übernehmen. ${ }^{42}$ Die Verfassung geht mithin davon aus, dass in aller Regel das Wohl des Kindes am besten durch die Eltern verwirklicht wird. ${ }^{43}$ Dies umfasse unter anderem »die Befugnis, die Lektüre der Kinder zu bestimmen «. ${ }^{44}$ Der in Art. 6 Abs. 2 u. 3 GG manifestierte Vorrang der Eltern im Sinne der Eigenständigkeit und Selbstverantwortlichkeit bei der Erziehung der Kinder begrenzt das gleichzeitig statuierte staatliche Wächteramt. ${ }^{45}$

Vor diesem Hintergrund sind Regelungen zu Jugendschutzprogrammen ( $§ 11$ JMStV), welche Eltern und sonstigen Personensorgeberechtigten die Möglichkeit geben, in Ausübung ihres elterlichen Erziehungsrechts selbst zu entscheiden, ob und welche beschränkenden Einstellungen sie für ihre Kinder vornehmen, hinsichtlich des Jugendschutzziels verfassungsrechtlich legitim. Demgegenüber schmälern gesetzlich vorgeschriebene Jugendschutz-Voreinstellungen die eigenständige Erziehungsentscheidung der Eltern, wenn z.B. durch 
technische Vorkonfigurationen »ab 18 «bereits einer elterlichen Entscheidung für das $\mathrm{Ob}$ und Wie einer Jugendschutzmaßnahme vorgegriffen wird. Sie partizipieren nicht am Verfassungsrang des staatlichen Jugendschutzauftrags. Dies ist entscheidend für die Frage, ob mit staatlichen Schutzmaßnahmen einhergehende Eingriffe in verfassungsrechtliche Grundfreiheiten insbesondere des Art. 5 Abs. 1 GG durch die Schranke des Jugendschutzes (Art. 5 Abs. 2 GG) gerechtfertigt werden können. ${ }^{46}$

\section{Schluss}

Ein technisches »Gottesteilchen«, das einen nur annähernd flächendeckenden, lückenlosen Jugendschutz gewährt, ist jedenfalls bei kursorischer Durchsicht der rechtsstaatlichen Demokratien zur Verfügung stehenden national-regulativen Mittel nicht in Sicht. Die vorstehenden Ausführungen werfen - ungeachtet der dargestellten rechtlichen und rechtstatsächlichen Probleme nationalgesetzlicher Regelungen zum technischen Jugendschutz - die eher rechtspolitische Frage auf, wie weit gesetzliche Regulierung auch im Bereich des technischen Jugendmedienschutzes überhaupt gehen darf und ob der Gesetzgeber nicht Entscheidungen - gerade im Bereich der 0/6/12/16/18 Angebotsinhalte in der häuslichen und mobilen Nutzersphäre - primär der elterlichen Erziehung überantworten kann und muss. Die wichtige Aufgabe der Förderung von Informations-, Medien- und auch Technikkompetenz mit Blick auf die Umsetzung technischer Jugendschutzlösungen durch Eltern bleibt hiervon freilich unberührt.

1 Vgl. nur BGH NJW 2008, 1882 ff. m. Anm. Engels/Jürgens = MMR 2008, 400 ff. m. Anm. Liesching und Anm. Waldenberger.

2 Vgl. zur gesetzlichen Einordnung unterschiedlicher Entwicklungsbeeinträchtigungsgrade nach Altersstufen $\S 5$ Abs. $1 \mathrm{JMStV}, \S 14$ Abs. 1 und 2 JuSchG; ausführl. Liesching/ Schuster, Jugendschutzrecht - Kommentar, 5. Aufl. 2011 zu den genannten Vorschriften.

3 JuSchRL vom 15.10.2019.

4 Vgl. schon BayLT-Drs 14/10246, S. 20.

5 Die Bejahung der Eignung von »JusProG durch die FSM wurde indes zwischenzeitlich in der KJM-Entscheidung v. 15.05.2019 als unvertretbar und als Überschreitung des FSM-Beurteilungsspielraums eingestuft, da Jugendschutzprogramme insbesondere einen plattform- und systemübergreifenden Schutz bieten müssten. Das VG Berlin hat die KJMBeurteilung als rechtswidrig angesehen (VG Berlin Beschl. v. 28.08.2019 - VG 27 L 164.19 MMR 2019, MMR 771 ff. m. Anm. Liesching). Die Bejahung der Eignung von JusProG ist mithin weiterhin rechtswirksam.

6 Vgl. Fn. 3 der KJM-JSP-Kriterien, Stand v. 12.10.2016; Keber, in: Bornemann/Erdemir, JMStV - Kommentar, 2017, § 11 Rn. 24.
7 Bayer. LT-Drs. 17/9700, 26.

8 Bayer. LT-Drs. 17/9700, 26. In der Amtl. Begründung wird dann gefordert, dass der Stand der Technik »jedenfalls diejenigen Schutzmaßnahmen « umfasse, »die als solche vom Jugendmedienschutz-Staatsvertrag vorgegeben werden «. Hiernach müsse das Jugendschutzprogramm »also technische Alterskennzeichnungen nach Altersstufen sowie auch solche technische Kennzeichnungen lesen können, die auf Sendezeitbeschränkungen hinweisen (»Label-Z«) « (Bayer. LTDrs. 17/9700, 26). Dies ist mit den eigenen gesetzlichen Vorgaben nicht vereinbar. Bei der Abfassung der Begründung ist offenbar bereits wieder vergessen worden, dass der zuvor gefasste Wortlaut des S. 3 das Erfordernis des Stands der Technik nur auf die Erkennungsleistung, hingegen gerade nicht auf das Auslesen von Kennzeichen erstreckt (missverständlich daher auch KJM-JSP-Kriterien, Stand v. 12.10.2016)

9 Vgl. nur jüngst BayVGH, Beschl. v. 01.09.2020 7 ZB 18.1183, Vorinstanz: VG München, Urt. v. 22.03.2018 - M 17 K 16.5590.

10 Richtlinie 2000/31/EG des Europäischen Parlaments und des Rates vom 8. Juni 2000 über bestimmte rechtliche Aspekte der Dienste der Informationsgesellschaft, insbesondere des elektronischen Geschäftsverkehrs, im Binnenmarkt (»Richtlinie über den elektronischen Geschäftsverkehr«), nachfolgend ECRL (ABl. Nr. L 178 S. 1).

11 EuGH, Urt. v. 25.10.2011 - C-509/09 und C-161/10, NJW 2012, 137, 141 Rn. 67 m. Anm. Brand, NJW 2012, 127 ff. In der Rechtsliteratur wird darauf hingewiesen, dass der EuGH insoweit die mit Art. 3 Abs. 1 und 2 ECRL bewirkte Einschränkung des nationalen Sachrechts bestätigt habe; vgl. Weber, MMR 2012, 45, $49 \mathrm{f}$.

$12 \mathrm{Zu}$ den Abweichungen im Rahmen der deutschen Umsetzung in § 3 TMG vgl. ausführl. Liesching, Das Herkunftslandprinzip der E-Commerce-Richtlinie und seine Auswirkung auf die aktuelle Mediengesetzgebung in Deutschland, 2020, S. $50 \mathrm{ff}$.

13 Vgl. Schemmer in: Epping/Hillgruber, BeckOK Grundgesetz, 43. Edition, Stand: 15.05.2020, Art. 5, Vor Rn. 1.

14 Jarass/Pieroth, GG - Kommentar, 16. Aufl. 2020, Art. 5 Rn. 27; Schemmer in: Epping/ Hillgruber, BeckOK Grundgesetz, 43. Edition, Stand: 15.05.2020, Art. 5 Rn. 34.

15 Vgl. BVerfG NJW 2012, 3423.

16 Vgl. BVerfG, ZUM-RD 2011, 137, 139; siehe auch BVerfGE 7, 198, 209; 77, 65, 74.

17 Siehe die Übersicht der von der KJM positiv bewerteten Konzepte technischer Mittel, https://www.kjm-online.de/fileadmin/ user_upload/KJM/Aufsicht/Technischer Jugendmedienschutz/Uebersicht_technische_ Mittel.pdf

18 Nach Auffassung des BVerfG haben gesetzliche Bestimmungen zum Schutze der Jugend, wenn sie die Freiheitsrechte des Art. 5 Abs. 1 GG einschränken, die Anforderungen an ein allgemeines Gesetz zu erfüllen; vgl. BVerfGE $124,300=$ NJW 2010, 47, 50.

19 Vgl. BVerfGE 117, 244, 260 = NJW 2007, 1117; BVerfG NJW 2012, 3423.

20 Vgl. BVerfGE 7, 198, 208 = NJW 1958, 257; BVerfGE $82,43,50=$ NJW 1990, 1980; BVerfG NJW 2012, 3423, st. Rspr.
21 Vgl. BVerfGE 71, 162, 181 = NJW 1986, 1533; BVerfGE 74, 297, 337 = NJW 1987, 2987; BVerfG NJW 2012, 3423.

22 Siehe hierzu unten Punkt VI.

23 Vgl. BVerfGE 24, 119, 135 u. 138 = NJW 1968, 2233.

24 Hoffmann/Volkmann in: Spindler/Schuster, Recht der elektronischen Medien, 4. Aufl. 2019, § 7 TMG Rn. 35.

25 Siehe die Übersicht der von der KJM positiv bewerteten Konzepte technischer Mittel, https://www.kjm-online.de/fileadmin/ user_upload/KJM/Aufsicht/Technischer_ Jugendmedienschutz/Uebersicht_technische_ Mittel.pdf

26 Vgl. zur Auslegung des Berichterstattungsprivilegs Altenhain, MMR-Aktuell 2010, 302778; Erdemir in: Bornemann/Erdemir, JMStV Kommentar, 1. Aufl. 2017 § 5 Rn. 76 ff.; Hopf, ZUM 2009, 191 ff.; Liesching, ZUM 2009, 367, $372 \mathrm{f}$.

27 Vgl. BT-Drs. 14/9013, S. 17; BVerfGE 11, 234, 237; BVerwGE 23, 112, 113; ausführl. Liesching, ZUM 2002, $868 \mathrm{ff}$.

28 BVerfGE 31, 113, 117; 22, 108, 212 f.; BVerwGE 19, 94, 96; 23, 112, 113; 85, 169, 176; VG Köln NJW 1987, 274, 275; BT-Drs. 14/9013, S. 17; Bandehzadeh, 2007, S. 89 ff.; Maunz/ Dürig, GG - Kommentar, Art. 74 Rn. 108; Beisel/Heinrich, NJW 1996, 491, 493; insoweit zustimmend: Landmann, NJW 1996, 3309 Meyer-Hesemann, DVBl. 1986, 1181, 1183; a.A. Reinwald, ZUM 2002, $119 \mathrm{ff}$.

29 BT-Drs. 14/9013, S. 17.

30 BT-Drs. 14/9013, S. 24 f.

31 Vgl. BVerfGE 98, 265 (299) = NJW 1999, 841.

32 Vgl. BVerfGE 98, 265 (299) = NJW 1999, 841, 842 f.; BVerfGE 67, 299, 324 = NJW 1985, 371 mwN.

33 BT-Drs. 14/9013, S. 13.

34 BT-Drs. 14/9013, S. 14.

35 Vgl. ausführl. Altenhain in: Löffler, Presserecht, 6. Aufl. 2015, § 1 JuSchG Rn. 28 ff.

36 Vgl. Bayer. LT-Drs. 14/10246, S. 13 f.

37 Im Verhältnis des Art. 5 GG zu Art. 12, 14 GG besteht indes regelmäßig ohnehin Idealkonkurrenz; vgl. Schemmer in: Epping/Hillgruber, BeckOK Grundgesetz, 43. Edition, Stand: 15.05.2020, Art. 5 Rn. $96 \mathrm{mwN}$.

38 Inhalts- und Schrankenbestimmungen im Rahmen des Art. 14 GG haben dem Grundsatz der Verhältnismäßigkeit zu genügen; BVerfGE 110, 1, 28 = NJW 2004, 2073, 2078. Zur Voraussetzung der Verhältnismäßigkeit von Beeinträchtigungen der Berufsfreiheit (Art. $12 \mathrm{GG}$ ) siehe BVerfGE 94, 372, $389 \mathrm{f}$. = NJW 1996, 3067; BVerfGE 102, 197, 213 = NVwZ 2001, 790; BVerfGE 103, 1, 10 = NJW 2001, 353; BVerfGE 106, 181, 191 f. $=$ NJW $2003,879$.

39 Siehe hierzu auch oben zu europarechtlichen Gesichtspunkten Punkt IV.2.

40 Vgl. Bayer. LT-Drs. 14/10246, S. 20.

41 Vgl. BVerfG NJW 1991, 1471, 1472.

42 Vgl. BVerfGE 24, 119, 150 = NJW 1968, 2233; BVerfGE 76, 1, 51 = NJW 1988, 626; BVerfGE 99, $145,156=$ NJW 1999, 631.

43 Vgl. BVerfGE 99, 216, 232 = NJW 1999, 557.

44 BVerfG NJW 1991, 1471, 1472 unter Verweis auf BVerfGE 7, 320, $323 \mathrm{f}$. = NJW 1958, 865.

45 Vgl. BVerfGE 24, 119, 135 u. $138=$ NJW 1968, 2233.

46 Siehe hierzu oben Punkt IV.3. 